Société d'histoire de la révolution de 1848 et des révolutions du XIXe siècle

$50 \mid 2015$

Sociétés et forces de sécurité au $\mathrm{XIX}^{\mathrm{e}}$ siècle

\title{
Bethel SALER, The Settlers' Empire. Colonialism and State Formation in America's Old Northwest
}

Philadelphia (Penn.), University of Pennsylvania Press, 2015, 392 p. ISBN : 978-0-8122-4663-6. 45 dollars.

\section{Tangi Villerbu}

\section{(2) OpenEdition}

Édition électronique

URL : http://journals.openedition.org/rh19/4893

DOI : $10.4000 /$ rh 19.4893

ISSN : $1777-5329$

Éditeur

La Société de 1848

Édition imprimée

Date de publication : 1 juillet 2015

Pagination : 244-246

ISSN : 1265-1354

\section{Référence électronique}

Tangi Villerbu, «Bethel SALER, The Settlers' Empire. Colonialism and State Formation in America's Old Northwest », Revue d'histoire du XIXe siècle [En ligne], 50 | 2015, mis en ligne le 01 juillet 2015, consulté le 25 septembre 2020. URL : http://journals.openedition.org/rh19/4893 ; DOI : https://doi.org/ $10.4000 /$ rh19.4893

Ce document a été généré automatiquement le 25 septembre 2020

Tous droits réservés 


\title{
Bethel SALER, The Settlers' Empire. Colonialism and State Formation in America's Old Northwest
}

\author{
Philadelphia (Penn.), University of Pennsylvania Press, 2015, 392 p. \\ ISBN : 978-0-8122-4663-6. 45 dollars.
}

\section{Tangi Villerbu}

\section{RÉFÉRENCE}

Bethel SALER, The Settlers' Empire. Colonialism and State Formation in America's Old Northwest, Philadelphia (Penn.), University of Pennsylvania Press, 2015, 392 p. ISBN :

978-0-8122-4663-6. 45 dollars.

1 L'histoire que raconte Bethel Saler dans cet ouvrage était a priori bien connue. L'ouest des Grands Lacs est depuis longtemps largement arpenté par les historiens, et d'importants ouvrages ont récemment apporté de précieux éclaircissement sur la période traitée dans The Settlers' Empire : les travaux de Lucy Eldersveld Murphy ou de Josh Hall ont par exemple donné à lire le récit complexe de la ruée sur le plomb, de Prairie du Chien ou de la Guerre de Black Hawk ${ }^{1}$. De même, aucun historien américain ne peut être surpris par la succession de thèmes abordés par Bethel Saler, des traités indiens à la fin du commerce des fourrures, de l'arrivée des missionnaires à l'histoire du métissage. Mais la preuve est faite, et brillamment, qu'il y avait encore à dire. Il n'est qu'à comparer la synthèse sur l'histoire de la Frontière dans le Wisconsin qu'avait donné Mark Wyman en 1998 avec le traitement par Bethel Saler de la même région entre la Guerre de 1812 et la naissance de l'État du Wisconsin en 1848 pour s'en convaincre: Mark Wyman - que Bethel Saler ne se donne d'ailleurs pas la peine de citer, ce qui est pour le moins curieux - produisait, sans toujours démériter, une trame linéaire classique d'une Frontière turnerienne en voie de disparition ${ }^{2}$. Bethel Saler, elle, achève son ouvrage sur une étude de la production par Turner, enfant du Wisconsin, de 
son hypothèse de la Frontière et pose un autre regard sur le même objet. En effet, elle préfère lire dans l'Ouest l'histoire de la formation de l'État, un État à la fois postcolonial puisqu'ayant pris son indépendance et colonial puisqu'imposant lui-même un ordre du même type sur des territoires contigus. Et l'auteur s'inscrit à plein dans un récit de la construction de l'État à l'époque de la Jeune République qui est aujourd'hui en renouveau, que ce soit par son aspect fisco-financier ${ }^{3}$ ou par la conquête et l'organisation de l'Ouest, donc par l'étude des Territoires avant qu'il n'y ait des États ${ }^{4}$.

2 Après deux chapitres destinés à inscrire le récit du futur Wisconsin dans la moyenne durée de la conquête et de la colonisation de l'Ouest par les États-Unis, donc à déterminer avec quels outils législatifs (au premier chef l'Ordonnance sur la terre de 1785 et celle du Nord-Ouest de 1787) ou culturels l'État prendra en charge le destin des terres entre le Mississippi et le lac Michigan, Bethel Saler livre une réflexion sur la politique des traités indiens avant de se pencher dans quatre chapitres passionnants sur l'économie, la mission religieuse, la famille et l'ordre politique. Ainsi, elle oppose deux systèmes économiques, l'un fondé sur la réciprocité et l'autre sur l'échange de produits commerciaux. Le premier caractériserait le temps des fourrures et du métissage, celui du monde co-construit par les Indiens et les francophones de la région. Le deuxième serait celui imposé par les États-Unis et fondé sur le marché : loin de répondre aux attentes des législateurs des années 1780, il signalerait plutôt la manifestation d'un capitalisme triomphant porté par les colons venus du Nord-Est, notamment avec la ruée vers le plomb à partir des années 1820. La distinction entre deux modèles a une valeur certaine, mais à condition de ne pas l'exagérer: le commerce de la fourrure est aussi un marché intégré à l'échelle atlantique, et, sans le dire franchement, Bethel Saler insère son raisonnement dans des débats historiographiques sur la nature même du marché et du capitalisme ${ }^{5}$ qu'elle aurait dû interroger plus avant.

3 Suivent des développements sur les missionnaires qui doivent leur richesse à l'entremêlement des œuvres catholiques et protestantes. Le travail en commun est-il possible, les directions sont-elles identiques? Il fallait que tous acceptent un « double fardeau »: convertir les Indiens d'une part, maintenir les colons dans la juste voie d'autre part. La différence se joue dans la définition donnée aux processus de christianisation et de civilisation, sur laquelle les protestants font preuve d'une rigidité nettement supérieure, alors même que le contexte de métissage de longue durée devait mener à des accommodements face à la souplesse permanente des configurations identitaires. Il faut ici noter que Bethel Saler manque de sources catholiques. Non qu'elles n'existent pas: il faut les chercher là où elles sont, dans les institutions catholiques aux États-Unis et en Europe, et non se contenter de quelques pièces publiées. Cela aurait permis à l'auteur d'aller plus loin dans ses réflexions sur l'aspect international des réseaux religieux mais aussi, par exemple, sur les efforts catholiques en faveur de la tempérance.

4 Un lien aurait dès lors pu être construit avec le chapitre suivant, qui concerne l'imposition de normes culturelles et s'appuie surtout sur l'analyse quantitative des décisions des premières cours de justice locales. Il appert que les juges ont insisté sur l'effacement de formes à leurs yeux inacceptables de concubinage et de rôles genrés pour imposer par la loi une culture unique du mariage et de la famille. Leur réussite est palpable mais il ne faut pas pour autant grossir le trait du refus et de l'opposition entre deux mondes: de ce point de vue l'analyse serrée du cas de l'enlèvement par une 
famille de colons, les Partridge, d'un enfant Menominee qu'ils affirmaient être le leur est riche d'enseignements. Bethel Saler termine sa réflexion sur la séquence 1846-1848 en étudiant parallèlement la difficile rédaction d'une constitution pour le nouvel État du Wisconsin et la création d'une structure étatique particulière, la Société historique $\mathrm{du}$ Wisconsin. Dans un contexte où les jeunes hommes blanc venus du Nord-Est et démocrates dominèrent largement les deux conventions constitutionnelles successives, les points les plus épineux de cette époque furent débattus : défense des petits colons propriétaires, vote des Africains-Américains et des Indiens, existence d'un système bancaire et de papier monnaie... tandis que commençait de s'écrire une histoire régionale balançant entre tentative d'un récit progressiste linéaire et surgissement inopiné de l'extrême complexité des processus en jeu.

5 Le propos est riche, touffu, et de ce fait propre à susciter le débat. Je ne citerai ici que deux axes de réflexion généraux. Il semble finalement que l'effort de formation de l'État, au cœur de l'ouvrage, mériterait d'être mieux circonscrit, que l'État lui-même devrait être l'objet d'une définition plus ferme qui permettrait d'y voir clair dans son rôle. Il est ici législateur, prescripteur, mais il s'incarne peu ou sans qu'il y ait de réflexion sur cette incarnation: qui construit l'État, finalement? Qui sont ces agents qui le mettent en scène et imposent sa force ? Bethel Saler en cite quelques-uns : le juge Doty, l'agent indien Nicholas Boilvin (qui a d'ailleurs le même profil culturel a priori que ceux à qui il doit imposer l'État, ce qui pose question)... Mais il manque un portrait de groupe, une étude sérielle de ceux qui font l'État, comme Samuel Watson en a réalisé pour les officiers de l'armée fédérale ${ }^{6}$ ou Catherine Cahill pour les agents indiens d'une époque plus tardive ${ }^{7}$. D'autre part, ce n'est qu'a posteriori que la chronologie paraît justifiée, et appuyée sur deux événements de l'ordre du politique: une guerre en amont, une constitution en aval. Cela pose évidemment une question cruciale qui hante les historiens américains depuis que Turner a énoncé son hypothèse : comment arrêter le récit de l'Ouest? Les tenants de la Nouvelle histoire de l'Ouest des années 1980, ou ceux des borderlands actuellement, tendent à rejeter l'idée même de rupture. Bethel Saler, elle, choisit d'en construire une, faisant fi de ce qui au-delà de 1848 participe encore de la même construction de l'État - elle écarte ainsi, sans même l'évoquer, l'industrie du bois, centrale dans le nord de l'État - mais en posant fortement que certains gestes, certains mots, closent des moments de l'histoire et en ouvrent d'autres. En l'occurrence, en redonnant une vie au moment territorial que chaque espace au-delà des Appalaches a connu, elle redonne vie à l'état dans l'histoire américaine.

\section{NOTES}

1. Lucy Eldersveld Murphy, A Gathering of Rivers. Indians, Métis, and Mining in the Western Great Lakes, 1737-1832, Lincoln (Nebr.), University of Nebraska Press, 2000 et Great Lakes Creoles. A FrenchIndian Community on the Northern Borderlands, Prairie du Chien, 1750-1860, New York, Cambridge University Press, 2014 (publié quelques semaines seulement avant The Settlers' Empire) ; Josh W. Hall, Uncommon Defense. Indian Allies in the Black Hawk War, Cambridge (Mass.), Harvard University Press, 2008. 
2. Mark Wyman, The Wisconsin Frontier, Bloomington (Ind.), Indiana University Press, 1998.

3. Max Edling, A Revolution in Favor of Government. Origins of the U.S. Constitution and the Making of the American State, New York, Oxford University Press, 2003 et A Hercules in the Cradle. War, Money, and the American State, 1783-1867, Chicago (Ill.), University of Chicago Press, 2014.

4. William H. Bergmann, The American National State and the Early West, New York, Cambridge University Press, 2014 ; John Van Atta, Securing the West. Politics, Public Lands, and the Fate of the Old Republic, 1785-1850, Baltimore (Mar.), Johns Hopkins University Press, 2014.

5. Le débat a été relancé récemment, $c f$. Seth Rockman, 'What Makes the History of Capitalism Newsworthy ?', Journal of the Early Republic, n³4/3 (automne 2014), p. 439-466.

6. Samuel J. Watson, Jackson's Sword. The Army Officer Corps on the American Frontier, 1810-1821, Lawrence (Kan.), University Press of Kansas, 2012 et Peacekeepers and Conquerors. The Army Officer Corps on the American Frontier, 1821-1846, Lawrence (Kans.), University Press of Kansas, 2013.

7. Cathleen D. Cahill, Federal Fathers and Mothers. A Social History of the United States Indian Service, $1869-1933$, Chapel Hill (C.N.), University of North Carolina Press, 2011. 